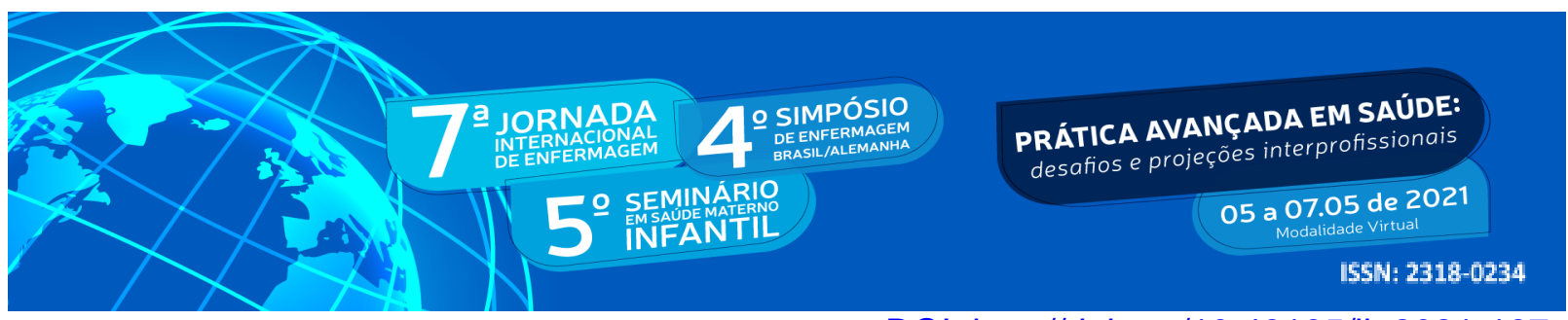

DOI: http://doi.org/10.48195/jie2021-187

\title{
QUANDO A SÍNDROME DE DOWN BATE À SUA PORTA: RELATO DE EXPERIÊNCIA ${ }^{1}$
}

\section{Rosani Viera Lunardi²; Pâmela Schultz Danzmann³ ${ }^{3}$ Luciane Najar Smeha ${ }^{4}$}

\begin{abstract}
RESUMO
Um dos momentos mais difíceis para a família é o da comunicação do diagnóstico e o processo de luto pela criança idealizada. Objetiva-se relatar a experiência de construção do documentário "Quando a síndrome de Down bate à sua porta", produto do Mestrado Profissional em Saúde Materno Infantil da Universidade Franciscana - UFN. Trata-se de um relato de experiência sobre a construção do documentário. Para isso foram entrevistadas 8 mães de crianças e adultos diagnosticados com SD, 4 profissionais da área da saúde como médicos pediatras e 1 psicóloga. Tanto os profissionais quanto as mulheres relataram momentos de tristeza, dor e luto frente à notícia do diagnóstico. Em síntese, o documentário aborda experiências mães e médicos no momento da comunicação do diagnóstico de síndrome de Down
\end{abstract}

Palavras-chave: Comunicação; Síndrome de Down; Maternidade; Profissionais da Saúde.

\begin{abstract}
One of the most difficult moments for the family is the receiving the diagnosis due to the mourning process of the perfect child. The objective is to report the experience of making the documentary "When Down's syndrome knocks on your door", a product of the Professional Master's Degree in Maternal and Child Health at Universidade Franciscana - UFN. This is an experience report on the making of the documentary. For this, 8 mothers of children and adults diagnosed with DS were interviewed, 4 health professionals as pediatricians and 1 psychologist. Both professionals and women reported moments of sadness, pain and mourning in the face of news of the diagnosis. In summary, the documentary addresses the experiences of mothers and doctors at the time of communicating the diagnosis of Down's syndrome
\end{abstract}

Key Words: Communication; Down Syndrome; Maternity; Health Professionals.

\footnotetext{
${ }^{1}$ Relato de Experiência do documentário "Quando a Síndrome de Down bate à sua porta".

${ }^{2}$ Mestre pelo Mestrado Profissional Saúde Materno Infantil da Universidade Francisca (UFN), Santa Maria/ RS/ Brasil.

3 Estudante do Curso de Curso de Psicologia da Universidade Franciscana (UFN), pamelapsicologia10@gmail.com - Santa Maria/RS/ Brasil.

${ }^{4}$ Orientadora e docente do Curso de Psicologia e do Mestrado Profissional Saúde Materno Infantil da Universidade Francisca (UFN), lucianenajar@yahoo.com.br - Santa Maria/ RS/ Brasil.
} 


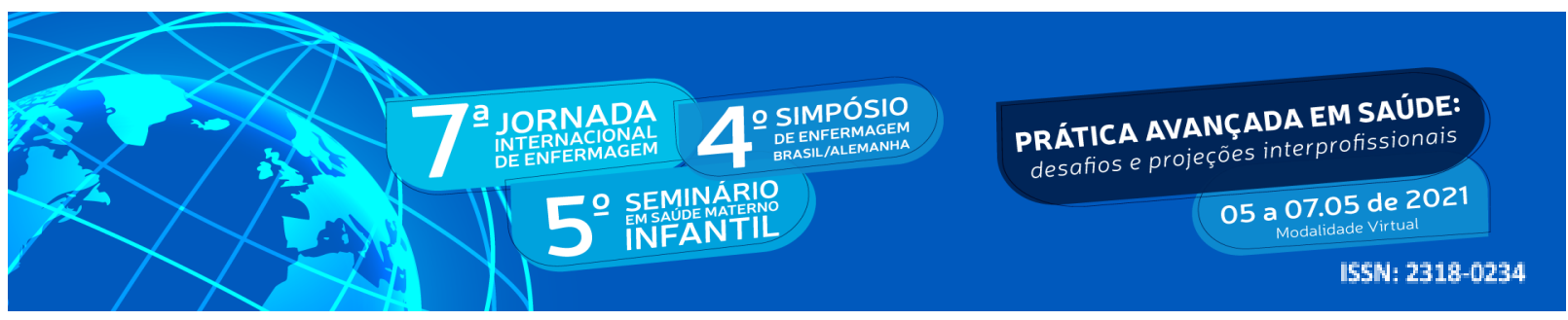

\section{INTRODUÇÃO}

O documentário é um instrumento importante porque pode ter a finalidade de enquadrar desafios dentro de uma sociedade. Muitas vezes a tecnologia de informação e linguagem audiovisual buscam explorar questões que podem não ser acessíveis à população em geral. O documentário "Quando a síndrome de down bate à porta" representa essas dificuldades e traz reflexões importantes no campo da maternidade de uma pessoa com Síndrome de Down (SD).

Posto isso, destaca-se que a SD caracteriza-se por ser uma síndrome genética causada pela trissomia do cromossomo 21. Isso significa que essas pessoas têm 47 cromossomos ao invés de 46. A idade materna, é uma das principais causas, já que a partir dos 35 anos, as chances de gerar uma criança com SD aumenta gradualmente. Outro ponto importante a salientar é que a SD não apresenta prevalência por classe social, gênero ou etnia (CROMWELL, 2014).

Dentre as características principais da síndrome encontra-se a braquicefalia, que se refere a um diâmetro fronto-occipital muito pequeno, fissuras palpebrais com inclinação superior, pregas epicânticas, base nasal achatada e hipoplasia da região mediana da face. Observa-se também na pessoa com SD pescoço curto e largo, mão e pés pequenos, apenas uma prega palmar, boca pequena e língua protuberante, olhos amendoados e rosto arredondado. Ademais, logo após o nascimento a criança tem dificuldade de sucção e deglutição. E também pode ocorrer um atraso no desenvolvimento de alguns reflexos do bebê (MARTINS et al., 2011).

A partir do momento em que a criança é exposta a linguagem ela vai assimilando os recursos linguísticos que lhes são oferecidos. Contudo, tratando-se de uma criança com SD, a linguagem também é prejudicada. Isso pode acontecer porque no início do desenvolvimento linguístico, a criança com SD parece responder menos aos estímulos verbais, bem como os estímulos não verbais como sorrisos e gestos. A dificuldade pragmática também pode ser citada nesse sentido, já que podem ter dificuldades em manter e respeitar o tempo de fala (LIMA; DELGADO; CAVALCANTE, 2017; SERRANO; IZUZQUIZA, 2018).

Contudo, as características fenotípicas e a dificuldade do desenvolvimento da linguagem podem ser menos dolorosas que o momento no qual os profissionais da saúde confirmam o diagnóstico. Isso pode não estar relacionado diretamente ao diagnóstico mas a 


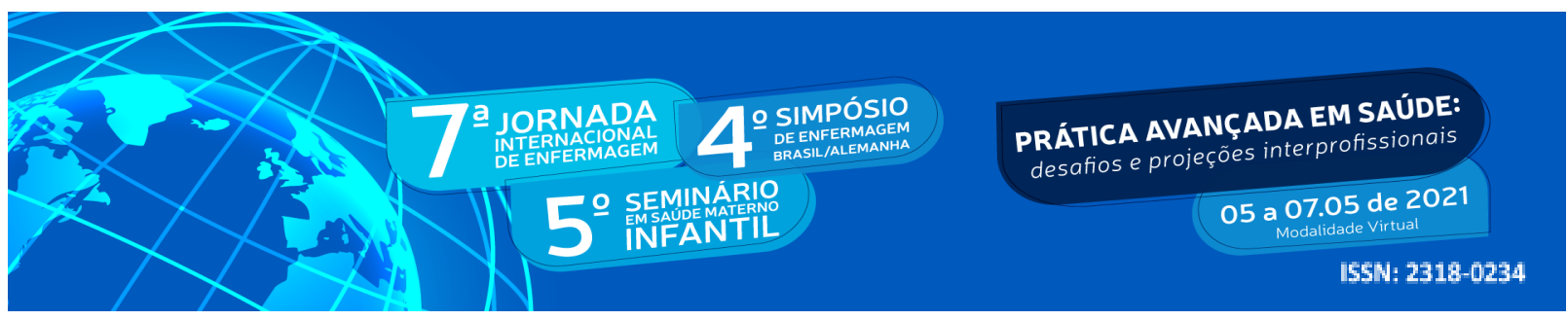

forma como se é notificado. Algumas vezes, o que acontece é que a SD é apresentada antes da criança à mãe e isso implica negativamente no processo de proximidade da mãe e o bebê (PEREIRA; ANDRADE; ALMEIDA, 2018; SILVA; GADELHA; CARVALHO, 2017).

O diagnóstico ou a suspeita, podem ser comunicados logo após o nascimento e desencadeia tensão, angústia, tristeza e estresse. Isso ocorre porque a mãe imaginou e desejou durante toda a gestação um(a) filho(a) saudável e perfeito. Esses sentimentos podem ser intensificados se o profissional não estiver preparado para diagnosticar e não esclarecer as dúvidas, medos e incertezas que surgem com a notícia (SUNELAITIS; ARRUDA; MACON, 2007).

O trabalho se justifica por ser uma tecnologia social que pode servir como um instrumento pedagógico para os cursos da saúde, utilizado para comunicação de diagnósticos e de reflexão dos profissionais da área de saúde, e que permite estabelecer melhorias nos futuros comunicados de diagnósticos.

\section{OBJETIVO}

Objetiva-se por meio deste trabalho relatar a experiência de construção do documentário "Quando a síndrome de Down bate à sua porta", produto do Mestrado Profissional em Saúde Materno Infantil da Universidade Franciscana - UFN.

\section{METODOLOGIA}

Trata-se de um relato de experiência que segundo Oliveira (2003) caracteriza-se como um texto que traz experiências importantes para a área de atuação. Ao narrar a experiência, salientamos as etapas metodológicas que foram utilizadas para a construção do documentário. Primeiramente foi realizada uma procura de publicações sobre o tema com vistas ao cenário da arte. Após, foram entrevistadas 8 mães de crianças e adultos diagnosticados com SD, 4 profissionais da área da saúde como médicos pediatras e 1 psicóloga. A coleta de dados ocorreu mediante questionário sociodemográfico e entrevista semiestruturada realizada individualmente, sete mães e quatro médicos na modalidade presencial, uma mãe e dois médicos por vídeo chamada, uma mãe e três médicos pelo áudio do WhatsApp devido ao 


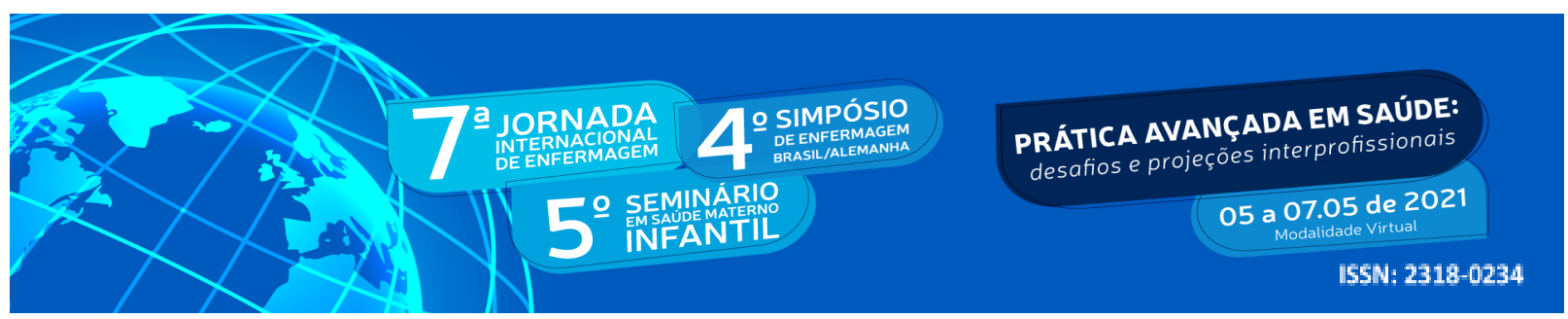

contexto da pandemia. A decupagem e edição foram realizadas pelas autoras com auxílio de um profissional da área do jornalismo.

\section{DESENVOLVIMENTO}

No que se refere à construção do documentário, destaca-se que o vídeo tem duração de 34 minutos e foi produto de Dissertação de Mestrado em Saúde Materno Infantil da Universidade Franciscana- UFN da egressa Rosani Lunardi. As entrevistas ocorreram no ano de 2020, nos meses de junho a outubro nas casas das mães ou no lugar escolhido pelas mesmas.

As seguintes perguntas nortearam as entrevistas das mães: Como foi informada do diagnóstico da SD? Como se sentiu ao receber a notícia do diagnóstico? Você gostaria que a comunicação do diagnóstico tivesse sido diferente? Por quem se sentiu apoiada no momento da notícia? Como você gostaria que fosse a experiência de mães que ainda terão um filho com SD? Já os médicos responderam: Como é feita a abordagem para comunicar a notícia do diagnóstico da SD? Qual o melhor momento para comunicar a notícia? Quais sentimentos das mães diante da notícia? Sente dificuldades nessa comunicação? Como aprendeu a comunicar notícias difíceis? As entrevistas foram realizadas em datas e locais escolhidos pelas mães ou médicos, com duração média de 30 minutos à $1 \mathrm{~h} 15 \mathrm{~min}$. Todos os participantes assinaram o Termo de Consentimento Livre e Esclarecido (TCLE) e uma autorização para veiculação de imagem.

Todas as perguntas se entrelaçam de alguma forma, notou-se que na visão materna a comunicação sobre o diagnóstico de SD pode ocorrer de várias formas, dependendo do profissional, da época e do preparo médico. Nesse sentido, uma mãe relatou que no seu caso, no momento de noticiar o diagnóstico foi empregado o termo "mongolismo", que hoje é ofensivo e ultrapassado. Isso se explica porque, no início, o quadro de síndrome de Down foi definido por Dr. John L. H. Down, como "mongolismo" porque as crianças européias possuíam semelhanças com a raça mongólica, como pálpebras inclinadas parecido com os asiáticos (SILVA; FERREIRA, 2008). Além disso, a mãe destaca que tratava-se de um tempo em que não era fácil o acesso à informação, pois não existia internet e nenhum tipo de 


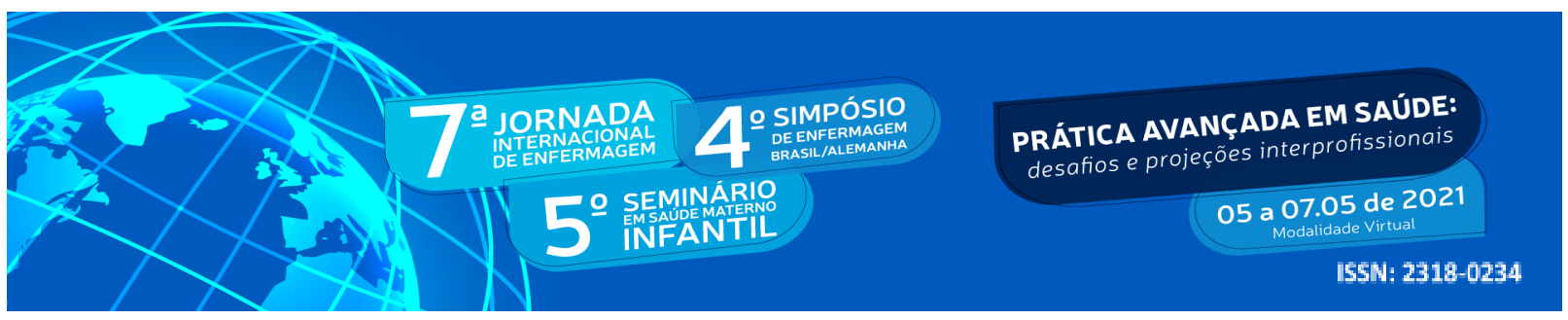

tecnologia de informação que facilitasse o conhecimento do diagnóstico assim como hoje. As fontes informativas eram revistas, jornais e livros.

Posto isso, destaca-se que hoje o termo atribuído para quem tem síndrome genética causada pela trissomia do cromossomo 21 é pessoa com SD (MOREIRA; HANI; GUSMÃO, 2000). Contudo, muitas vezes a forma como se noticia a síndrome ainda é fria e sem informações (ÁVILA et al., 2017). Isso gera sentimentos de incerteza, tristeza, insegurança e desorganização após saberem que o(a) filho(a) possui deficiência, então quanto maior o apoio aos pais, mais seguros estarão para cuidarem de seus filhos (STORT et al., 2018). As mães relatam terem vivenciado momentos de tristeza, dor e luto. Além disso, para elas é muito importante que outras mulheres não passem pela mesma situação, Para isso é necessário profissionalismo, e isso se define a partir do momento que o profissional tem carinho, empatia e compreensão ao dar uma notícia.

Uma das mães participantes referiu o que podia ser diferente na sua experiência. Ela não foi comunicada nos primeiros momentos e que os profissionais demoraram muito para falar de suas suspeitas devido acreditarem que ela poderia não aceitar muito bem a notícia e isso afetá-la profundamente. Diz que foi muito mais impactante ser comunicada muito tempo depois. A maioria das mães partilharam desse sentimento, pois perceberam diferenças importantes nos seus filhos assim que nasceram, sendo que algumas incomodam-se com a demora da comunicação. Para essas mulheres existem médicos bons, mas não são todos.

Uma pesquisa realizada por Luizada et al. (2015) relata que a maioria dos profissionais só comunica a notícia de deficiência depois da aproximação de pais e filho(a), pois justificam não ser a hora do nascimento o momento adequado. Isso não significa que as mães não sofreram com a notícia. As mulheres do documentário ficaram abaladas, mas aos poucos foram absorvendo a realidade e aceitando o(a) seu(ua) filho(a).

A comunicação, na percepção de algumas mães, é relatada pela própria criança. A mãe M6 diz que ao ficar sozinha nos primeiros momentos com a criança a mesma noticiou ser portadora da SD, pois a criança colocou a língua para fora e assim ela conseguiu focar em seu rosto e no pescoço e nas demais características da SD. A reação materna foi acolher, aceitar e conversar com a criança naquele momento. 


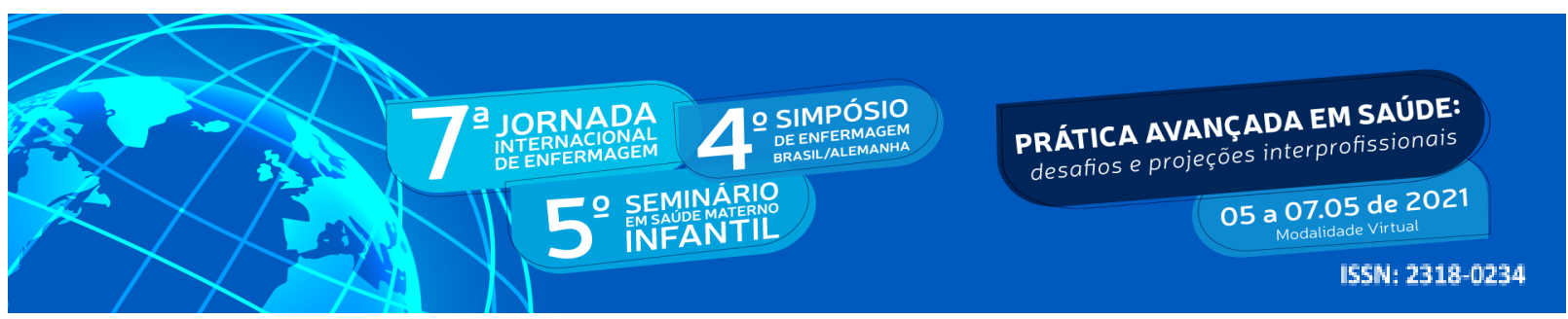

Destaca-se que é de suma importância o apoio psicológico logo após o diagnóstico, porque isso pode facilitar o vínculo pais-bebê e também a aceitação da deficiência do filho (ÁVILA et al., 2018). Ademais, os pais devem ser acolhidos e terem suas dúvidas e incertezas minimizadas, nesse sentido é necessário orientações aos pais para prepará-los para o cuidado do(a) filho(a) com SD (ROSS; COSTA;PISSAIS, 2019). Na visão de algumas mães o apoio familiar e profissional é fundamental nesse momento. Contudo nem todas as mães puderam sentir a segurança de ter esse acolhimento inicial, principalmente por partir do contexto da saúde.

No que se refere a percepção dos profisssionais da saúde, um ponto importante a ser destacado na hora de comunicar o diagnóstico é que tanto as mães que no pré-natal souberam que o bebê poderia nascer com diagnóstico de SD, quanto as mães que descobrira na hora do nascimento que seu(a) filho(a) era portador da síndrome passaram por momentos de tristeza, dor e luto do(a) filho(a) imaginado(a). Segundo os pediatras, esse processo ocorre porque os pais criam esperança de que as suspeitas médicas podem estar erradas. $\mathrm{Na}$ visão dos profissionais, a comunicação pode ser um pouco mais fácil para aqueles pais que já foram comunicados da possibilidade ou detecção de SD durante a gestação. E não pode ser noticiado imediatamente, sem a mãe ter estabelecido vínculo com o filho(a), pois isso pode dificultar a relação mãe e bebê.

Segundo Kroff, Maia e Lima (2000) o que mais repercute emocionalmente nos pais não como se noticia a SD, mas também o processo de elaboração do luto, a gravidade da síndrome, a paridade do casal, além do próprio diagnóstico, prognóstico, o tipo de cuidado que vai ser necessário. Ademais, o momento do diagnóstico da SD deve ser tratado com muita cautela, devido os pais se defrontam com diversas emoções (ÁVILA, et al., 2018).

Para nenhum dos profissionais de saúde, a comunicação sobre a SD é um processo fácil. Até mesmo para aqueles profissionais experientes, já que ali estão sinalizando ou até confirmando que a criança possa ter dificuldades importantes em vários contextos da vida e necessitam da presença constante dos pais para superar diversos obstáculos. Ademais, os profissionais relatam que tiveram de aprender na prática como noticiar situações delicadas, já que as faculdades não aprofundam esse cenário, então isso passa ser um desafio no campo 


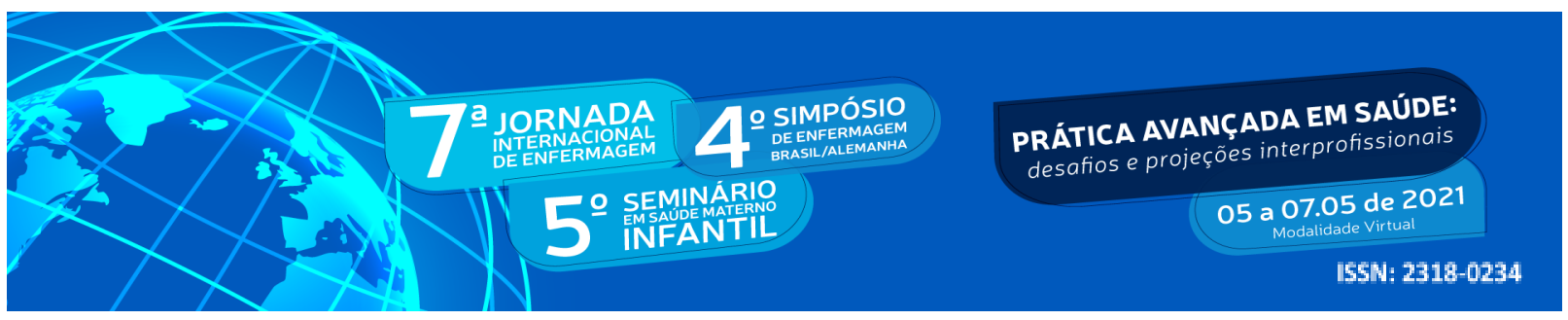

prático da medicina. O tempo e os erros são citados como principais aprendizados nesse sentido.

Em linhas gerais, a comunicação do diagnóstico de SD não é um processo fácil na percepção materna e médica. Os próprios profissionais da saúde salientam que há algumas décadas atrás o diagnóstico era apenas transmitido, sem muitas informações. Outras vezes a comunicação era imediata, logo após o nascimento da criança e aos poucos pode-se perceber que essa ação interferia no processo de vinculação de mãe e filho. Nesse sentido, conforme Luizada et al., (2015) os médicos podem se sentir despreparados e com dificuldades de dar a notícia, isso pode estar ligado diretamente a formação acadêmica que não prepara o profissional para esses momentos.

\section{CONCLUSÃO}

O documentário apresenta que o recebimento do diagnóstico de SD é um momento doloroso para as mães. Dessa forma, sentimentos de insegurança, medo, tristeza e luto são muito comuns. Tudo isso pode ainda ser mais difícil, caso o médico não tenha habilidade para fazer a comunicação.Os médicos ressaltam a importância de comunicar a deficiência no momento certo, o que preferem não informar no momento do parto, possibilitando que a mãe conheça o bebê e possa amamentar a primeira vez, antes de receber o diagnóstico, pois isso favorece vínculo-bebê.

Dessa forma destaca-se que por ser um momento delicado na vida das mães, é necessário empatia por parte dos profissionais da saúde, bem como preparo no campo acadêmico para que possam futuramente atuar com mais tranquilidade. Sugere-se mais estudos sobre o processo de comunicação de deficiências para as mães e os seus impactos nas famílias a partir desse momento.

Compreende-se que o documentário é uma tecnologia social muito potente para estudantes da área da saúde na graduação e pós-graduação. Ele também é uma ferramenta para associação de pais, para os pais e seus familiares, pode ser utilizado como estratégia metodológica em cursos de capacitação e educação continuada . 


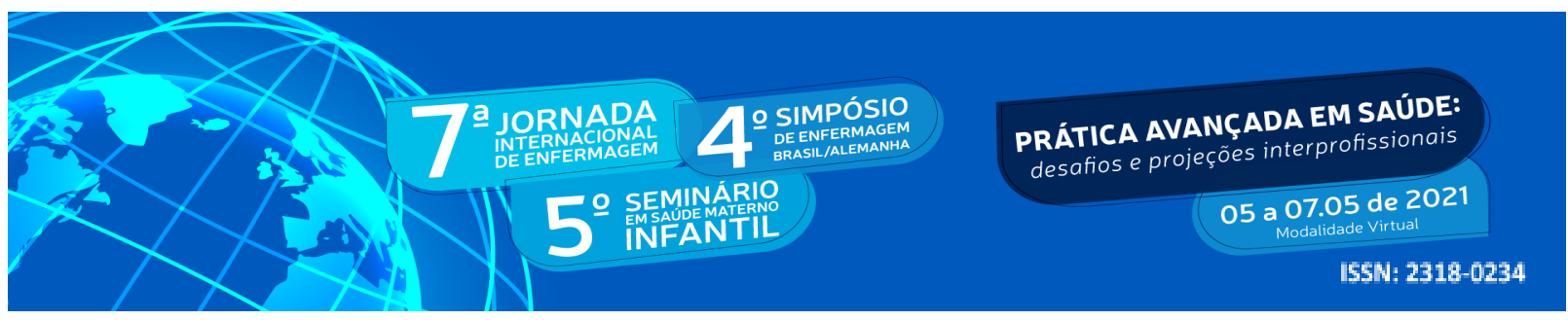

\section{REFERÊNCIAS}

ÁVILA, R.F. et al. Síndrome de Down: O impacto do diagnóstico sob a ótica dos pais, um projeto de extensão. Revista Fragmentos de Cultura - Revista Interdisciplinar de Ciências Humanas, v. 27, n. 1, p. 124-133, abr. 2017. Disponível em: http://dx.doi.org/10.18224/frag.v27i1.5448. Acesso: 13 mar. 2021.

CROMWELL, I. D.P. Clinical Practice Guidelines for Management of Children With Down Syndrome: Part I. J Pediatr Health Care, n. 28, v. 1, p. 105-110, 2014. Disponível em: http://www.scielo.org.mx/scielo.php?script=sci nlinks\&pid=S0186-23912016000500289000 02\&lng=en>. Acesso: 13 mar. 2021.

LIMA, I. L. B.; DELGADO, I. C.; CAVALCANTE, M. C.B. Desenvolvimento da linguagem na síndrome de Down: análise da literatura. Distúrbios da comunicação, n 29, v.2, p. 1-7. 2017. Disponível em: https://doi.org/10.23925/2176-2724.2017v29i2p354-364. Acesso: 13 mar. 2021.

KROEFF, C., MAIA, C.; LIMA, C. O luto do filho malformado. Femina, v. 28, p. 395-396, 2000 .

LUIZADA, V. el al. Experiências de médicos ao comunicarem o diagnóstico da deficiência de bebês aos pais. Ciência e Saúde, v.8, n.3, p.121-128,2015. Disponível em: http://dx.doi.org/10.15448/1983-652X.2015.3.21769. Acesso: 13 mar. 2021.

MARTINS, M. R. I et al. Avaliação das habilidades funcionais e de auto cuidado de indivíduos com Síndrome de Down pertencentes a uma oficina Terapêutica. Rev. CEFAC, São Paulo, 2011. Disponível em: $<$ http://www.scielo.br/pdf/rcefac/2012nahead/160-11.pdf Acesso: 13mar. 2021.

MOREIRA, L. M. A.; EL-HANI, C. N.; GUSMAO, F. A. F. A síndrome de Down e sua patogênese: considerações sobre o determinismo genético. Rev. Bras. Psiquiatr., São Paulo, v. 22, n. 2, p. 96-99, June 2000. Available from $<$ http://www.scielo.br/scielo.php?script=sci arttext\&pid=S1516-44462000000200011\&lng=e $\underline{\text { n \&nrm=iso }>\text {. }}$.

Access on 13 Mar. 2021. https://doi.org/10.1590/S1516-44462000000200011.

OLIVEIRA, F. M. A configuração textual da seção de metodologia em artigos acadêmicos de Lingüística Aplicada. 2003. 134f. Dissertação (Mestrado em Letras) - Universidade Federal de Santa Maria, Santa Maria, 2003.

PEREIRA, N. L.; ANDRADE, J. C. M. ; ALMEIDA, B. R. . Famílias e síndrome de Down: Estresse, coping e recursos familiares. Psic.: Teor. e Pesq., Brasília, v. 34, e3445, 2018. .Disponível em: 


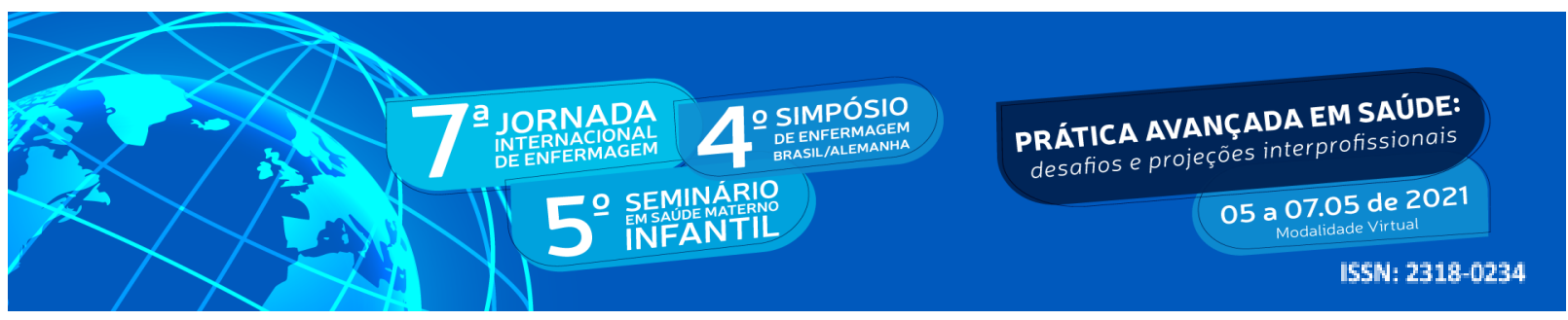

$<$ http://www.scielo.br/scielo.php?script=sci arttext\&pid=S0102-37722018000100504\&lng=e $\underline{\text { n\&nrm }=\text { iso }>\text {. }}$.

Access on 10 Mar. 2021. https://doi.org/10.1590/0102.3772e3445.

ROOS, L.; COSTA, A. E. K. DA.; PISSAIA, L. F.. Síndrome de Down: trajetórias do seu diagnóstico para os pais. Revista Interdisciplinar de Promoção da Saúde, Santa Cruz do Sul, v. 2, n. 1, p. 8-13, ago. 2019. Disponível em:https://doi.org/10.17058/rips.v2i1.13192. Acesso: 13 mar. 2021.

SERRANO, F., L.; IZUZQUIZA, D. Percepciones parentales sobre el impacto del síndrome de Down en la familia. Siglo Cero, v. 48, n. 2, p. 81-98, 23 feb.2018 Disponível em:https://doi.org/10.14201/scero20174828198. Acesso: 13 mar. 2021.

SILVA F. J. A. DA.; GADELHA, M. DO S. N.; CARVALHO, S. M. C. R. DE. S. Síndrome De Down: Reação das Mães Frente à Notícia e a Repercussão na Intervenção Fisioterapêutica da Criança. Revista Brasileira de Ciências da Saúde, v. 21, n. 2, p. 157-164, 11 mar. 2017.

SILVA, D. R. DA; FERREIRA, J. S. Influence of Physical Education on children affected by down syndrome. Journal of Physical Education, v. 12, n. 1, p. 69-76, 6 Jun. 2008. Disponível em: <http://periodicos.uem.br/ojs/index.php/RevEducFis/article/view/3784>. Acesso: 13 mar. 2021.

SUNELAITIS, R. C. ; ARRUDA, D. C. ; MARCOM, S. S.. A repercussão de um diagnóstico de síndrome de Down no cotidiano familiar: perspectiva da mãe. Acta paul. enferm., São Paulo , v. 20, n. 3, p. 264-271, Sept. 2007 . Disponível em: $:<$ http://www.scielo.br/scielo.php?script=sci arttext\&pid=S0103-21002007000300004\&lng= en\&nrm=iso $>$.

Acesso em: 10 Mar. 2021. https://doi.org/10.1590/S0103-21002007000300004.

STORT, A. H. et al. Parentalidade e Síndrome de Down: uma perspectiva dos pais. Revista Atas, v.2, p. 1312-1327. $2018 . \quad$ Disponível em: $<$ https://www.proceedings.ciaiq.org/index.php/ciaiq2018/article/view/1913/1863>. Acesso: 13 mar. 2021. 